\title{
A mitotic topoisomerase II checkpoint in budding yeast is required for genome stability but acts independently of Pds1/securin
}

\author{
Catherine A. Andrews, ${ }^{1,3}$ Amit C. Vas, ${ }^{1,3}$ Brian Meier,,${ }^{1,3}$ Juan F. Giménez-Abián,,${ }^{1,2}$ \\ Laura A. Díaz-Martínez, ${ }^{1}$ Julie Green, ${ }^{1}$ Stacy L. Erickson, ${ }^{1}$ Kristyn E. VanderWaal, ${ }^{1}$ Wei-Shan Hsu, ${ }^{1}$ \\ and Duncan J. Clarke ${ }^{1,4}$ \\ ${ }^{1}$ Department of Genetics, Cell Biology and Development, University of Minnesota Medical School, Minneapolis, Minnesota \\ 55455, USA; ${ }^{2}$ Proliferación Celular, Centro de Investigaciones Biológicas, 28040 Madrid, Spain
}

\begin{abstract}
Topoisomerase II (Topo II) performs topological modifications on double-stranded DNA molecules that are essential for chromosome condensation, resolution, and segregation. In mammals, G2 and metaphase cell cycle delays induced by Topo II poisons have been proposed to be the result of checkpoint activation in response to the catenation state of DNA. However, the apparent lack of such controls in model organisms has excluded genetic proof that Topo II checkpoints exist and are separable from the conventional DNA damage checkpoint controls. But here, we define a Topo II-dependent G2/M checkpoint in a genetically amenable eukaryote, budding yeast, and demonstrate that this checkpoint enhances cell survival. Conversely, a lack of the checkpoint results in aneuploidy. Neither DNA damage-responsive pathways nor Pds1/securin are needed for this checkpoint. Unusually, spindle assembly checkpoint components are required for the Topo II checkpoint, but checkpoint activation is not the result of failed chromosome biorientation or a lack of spindle tension. Thus, compromised Topo II function activates a yeast checkpoint system that operates by a novel mechanism.
\end{abstract}

[Keywords: Topoisomerase II; Top2; mitotic checkpoint; catenation; Mad2; Pds1]

Supplemental material is available at http://www.genesdev.org.

Received August 22, 2005; revised version accepted February 22, 2006.

Type-II DNA topoisomerases are essential conserved enzymes (DiNardo et al. 1984; Uemura and Yanagida 1984; Holm et al. 1985) abundant in interphase nuclei and major components of mitotic chromosomes (Earnshaw et al. 1985; Hirano and Mitchison 1991; Giménez-Abián et al. 1995). Topoisomerase II (Topo II) homodimers perform an ATP-dependent "strand-passage" reaction in which one double-stranded DNA (dsDNA) molecule is transported through a second, transiently cut dsDNA molecule. This unique cycle of DNA breakage, transport, and religation, which reversibly decatenates dsDNA and modulates supercoiling, is essential for mitosis (Yanagida and Wang 1987). It prepares chromosomes for segregation by unknotting all of the DNA molecules in the cell that inevitably became concatenated as a consequence of their replication (Cook 1991).

\footnotetext{
${ }^{3}$ These authors contributed equally to this work.

${ }^{4}$ Corresponding author.

E-MAIL Duncan.J.Clarke-2@umn.edu; FAX (612) 6266140.

Article and publication are at http://www.genesdev.org/cgi/doi/10.1101/ gad.1367206.
}

Decatenation must be coordinated with cell cycle progression. In G2 and early mitosis, removal of intra- and interchromosomal catenations allows chromosome individualization (Giménez-Abián et al. 2000) and condensation (Newport 1987; Charron and Hancock 1990; Wood and Earnshaw 1990; Adachi et al. 1991; Hirano and Mitchison 1991; Giménez-Abián et al. 1995; GiménezAbián and Clarke 2003). From the end of prophase, further decatenation allows sisters to become resolved, then segregate from one another in anaphase (Sundin and Varshavsky 1981; Wasserman and Cozzarelli 1986; Shamu and Murray 1992; Clarke et al. 1993; Downes et al. 1994; Giménez-Abián et al. 1995).

Not surprisingly, cell cycle progression in the absence of Topo II results in mitotic catastrophe. Thus, it has been proposed that biochemical surveillance systems, or checkpoints, control mitosis in response to the decatenatory activity of Topo II (Downes et al. 1994; GimenezAbian et al. 2002). Checkpoints monitor the progress of particular cellular processes (e.g., DNA replication), then send signals that restrain the cell cycle machinery and 
thereby inhibit the transition to the next cell cycle stage until these processes are complete (Clarke and GiménezAbián 2000). In this way, the cell cycle proceeds by an ordered series of processes. However, checkpoints are rarely so robust as to maintain cell cycle arrest. Rather, checkpoints are typically transient cell cycle delays that aim to allow some extra time for the process being monitored to become complete.

Treating mammalian G2 cells with Topo II inhibitors delays entry into mitosis (Kalwinsky et al. 1983) and checkpoint-evading agents such as caffeine bypass these delays (Downes et al. 1994). Thus, it was reasoned that this effect was due to a G2 checkpoint system that monitors Topo II activity or the catenation state of DNA. This putative G2 checkpoint was proposed to be distinct from the G2 DNA damage checkpoint, because the Topo II inhibitors used, such as ICRF-193, induced G2 delays without directly causing DNA strand breaks (Creighton and Birnie 1969; Tanabe et al. 1991; Downes et al. 1994). However, recent studies did detect DNA damage induced by these drugs, most likely generated indirectly as a consequence of interrupted decatenation, indicating that an overlap between G2 damage and Topo II checkpoints may exist (Dominguez et al. 2001; Mikhailov et al. 2002; Hajji et al. 2003; Adachi et al. 2004). Other work has implicated DNA damage response proteins (ATR, Brca1, and Ku) (Deming et al. 2001; Munoz et al. 2001) and the p38 pathway (Mikhailov et al. 2004) in Topo II checkpoint function. The Topo II inhibitor ICRF-193 has also been reported to delay the cell cycle at the metaphase-to-anaphase transition (Mikhailov et al. 2002; Skoufias et al. 2004), but whether this delay is due to Topo II inhibition (Skoufias et al. 2004) or to DNA breakage (Mikhailov et al. 2002) remains unresolved.

The cellular pathways that enforce these G2 and metaphase cell cycle delays have not been defined rigorously in genetic terms. Other eukaryotic checkpoint controls, the G1 and G2 DNA damage and the spindle assembly checkpoints, have been proven to exist by genetic means and have been rapidly characterized owing to their detailed study in lower eukaryotes, primarily yeasts. In budding and fission yeast, the essential role of Topo II in decatenating sister chromatids before anaphase has been demonstrated by means of temperature-sensitive top2 mutants (DiNardo et al. 1984; Uemura and Yanagida 1984; Holm et al. 1985; Uemura et al. 1986). However, at the nonpermissive temperature, top 2 cells have been reported to progress through mitosis without delay. In fact, the apparent lack of a Topo II checkpoint in any model system has impeded an exhaustive characterization of this critical checkpoint.

We sought genetic proof that Topo II checkpoints function to protect the genome, and report here the identification of a Topo II checkpoint in budding yeast. We have characterized top2 mutant strains that delay cell cycle progression such that G2/M lasts over three times its normal duration. As expected, a failure of this checkpoint response causes aneuploidy and reduces cell viability, but most interesting is that this Topo II checkpoint enforces its G2/M delay via an entirely unexpected mechanism. The checkpoint response does not rely on DNA damage checkpoint components, but rather it uses a subset of the spindle checkpoint proteins. However, the Topo II checkpoint is distinct from the conventional spindle checkpoint in two important ways; firstly, it is not activated in response to spindle damage or a lack of chromosome biorientation/tension, and secondly, it does not act by Pds1-dependent inhibition of Esp1/separase. Thus, the Topo II-dependent checkpoint defined herein is distinct from the known checkpoint systems.

\section{Results}

G2/M delay in top2-B44, a hypomorphic mutant of TOP2

Evidence that budding yeast cells lack a Topo II-dependent checkpoint comes from studies using the top2-4 allele that fails to delay mitosis at the nonpermissive temperature. Still, there are cases where mutations cause a "checkpoint-active" situation to arise, but simultaneously render checkpoint signaling ineffectual. One example is the lack of a DNA replication checkpoint signal in mutants that prevent replication origin firing. Based on this premise, we isolated new temperature-sensitive TOP2 alleles and assayed whether these mutations induced a cell cycle delay. Cells were released from G1 synchrony induced by mating pheromone, then samples were taken at intervals to score budding, spindle morphologies, and for FACScan analysis of DNA content. In wild-type cells, the interval between spindle assembly and anaphase (spindle elongation) was $14.8 \pm 3.2$ min ( $n=14$ experiments) (Fig. 1A; see Materials and Methods for calculations). Since spindle assembly is approximately coincident with the completion of DNA replication, we used the spindle assembly-to-spindle elongation interval as an estimate of the length of G2/M phase. (Note that in budding yeast, cell cycle stages between G2 and metaphase are indistinguishable cytologically, because the chromosomes become bioriented-the classical definition of metaphase-around the time that DNA replication is completed.)

In the top2 mutants described herein, budding, spindle assembly, and DNA replication occurred with kinetics indistinguishable from wild type (Fig. 1; data not shown). As previously reported, top2-4 mutants did not delay anaphase at the nonpermissive temperature of $32^{\circ} \mathrm{C}$ (Fig. 1A), but spindle elongation was blocked by expression of nondegradable securin (Clarke et al. 2001), Pds1, confirming that spindle elongation could be equated with anaphase in these cells (Supplementary Fig. 1). Remarkably, however, among the new mutant alleles of TOP2 that we isolated, G2/M delays were observed (Fig. 1A; Supplementary Fig. 2). For consistency, each mutant was assayed at $32^{\circ} \mathrm{C}$ (though most of the mutants tested were inviable at higher temperatures). The length of G2/M in one such mutant possessing a F977L substitution, top2$B 44$, was studied in detail and found to be $46.2 \pm 5.4 \mathrm{~min}$ ( $n=16$ experiments) (Fig. 1A), $>300 \%$ of the wild-type $\mathrm{G} 2 / \mathrm{M}$ period. In wild-type cells with large buds, the mi- 
Andrews et al.

G2/M delay in top2-B44 mutant

A

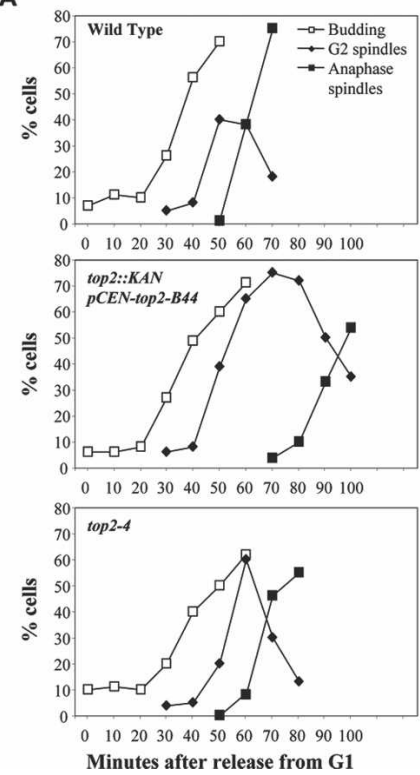

B
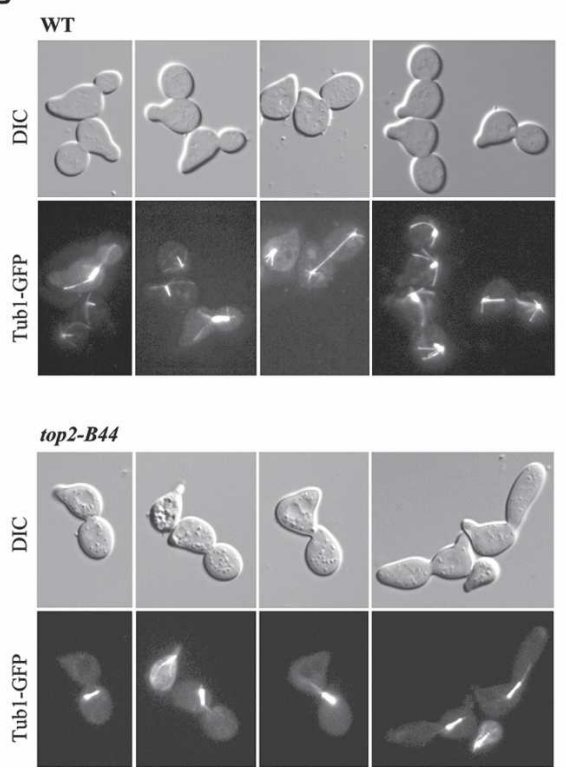

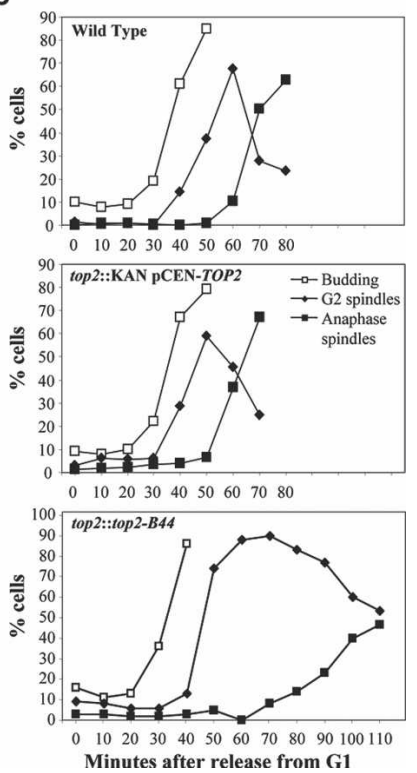

Figure 1. top2-B44 mutant cells delay in G2/M. Cell cycle analysis of wild-type versus top2 mutants at $32^{\circ} \mathrm{C}$, released from mating pheromone-induced G1 synchrony. Samples were processed for FACScan analysis of DNA content (not shown) and cytology (budding and spindle morphology was scored). (A) Cell cycle progression after release from G1 in wild-type versus top2::KAN pCEN-top2-B44 and top2-4. (B) Photomicrographs showing G2/M delay in top2::KAN pCEN-top2-B44 cells; wild-type cells with large buds (top; photos taken 70 min after release from G1) have elongated or disassembled spindles, while top2-B44 cells with large buds often contain short G2 spindles (photos taken 80 or 90 min after release from G1). (C) Cell cycle progression after release from G1 in wild-type versus a top2-null strain containing pCEN-TOP2(TRP1) (top two graphs) and in top2::top2-B44 cells (bottom graph).

totic spindle had invariably either elongated (anaphase) or had recently disassembled (telophase) (Fig. 1B; top). However, in top2-B44 mutants, the G2/M delay manifested as cells with large buds and short G2 spindles (Fig. $1 \mathrm{~B}$, bottom).

The top2 strains that we generated contained CEN plasmid-borne top2 alleles, covering a deletion of the endogenous TOP2 gene (i.e., top 2::KAN pCEN-top2-B44). Therefore, we sought to establish whether the presence of the CEN plasmid had an effect on the length of G2/M. In a strain containing the CEN plasmid harboring the wild-type TOP2 gene (Fig. $1 \mathrm{C}$ ), the $\mathrm{G} 2 / \mathrm{M}$ period at $32^{\circ} \mathrm{C}$ was consistent with that of cells not containing a CEN plasmid. Moreover, strains in which the top2-B44 allele was integrated at the endogenous locus (replacing the wild-type TOP2 allele; top2::top2-B44) or at the URA3 locus (in strains where endogenous TOP2 was deleted), similar G2 delays were observed (Fig. 1C, bottom graph). Therefore, the G2/M delay resulted from the top2-B44 mutation rather than the presence of the CEN plasmid. We conclude that top2 mutations can result in a substantial lengthening of the G2/M cycle phase, consistent with a checkpoint response being triggered as a result of perturbed Topo II function.

\section{G2/M delay in top2-B44 is DNA damage checkpoint independent}

In mammalian cells, whether Topo II checkpoints are unique from DNA damage checkpoint controls is a mat- ter of controversy. Drugs that inhibit Topo II without inducing cleavable complex formation, and therefore cannot induce DNA damage directly, nevertheless produce G2 and metaphase delays. But, it remains a possibility that some dsDNA breaks are induced indirectly as a consequence of Topo II inhibition, thus activating established DNA damage checkpoint controls.

To unequivocally answer this question, we asked whether top2-B44 mutants require DNA damage checkpoint signaling to enforce the G2/M delay (Fig. 2). Using the same cell cycle analysis as that described in Figure 1, we observed that top2-B44 and top2-B44 rad53-1 cells, the latter containing a checkpoint-null allele of the damage checkpoint kinase Rad53/Chk2, behaved identically, delaying in G2/M for $\sim 45 \mathrm{~min}$ (Fig. 2A). Moreover, Rad53 protein from top2-B44 cells did not undergo a robust phosphorylation up-shift on SDS-PAGE gels that would have been characteristic of DNA damage checkpoint activation (although a minor fraction of Rad53 did appear to have a reduced electrophoretic mobility; Fig. 2B). Similarly, top2-B44 mec1-1 cells, null for Mec1-dependent damage checkpoint function, delayed in G2/M before initiating mitosis; though in this case, our inability to synchronize these cells efficiently in G1 made it difficult to determine the extent of the delay (Fig. 2C). However, large budded cells with short G2 spindles were frequently observed in this strain (data not shown).

Lastly, we measured DNA damage in top2-B44 cells by counting Rad52 foci that form at sites of dsDNA breakage (Lisby et al. 2003). Rad52 foci were present at similar 
A
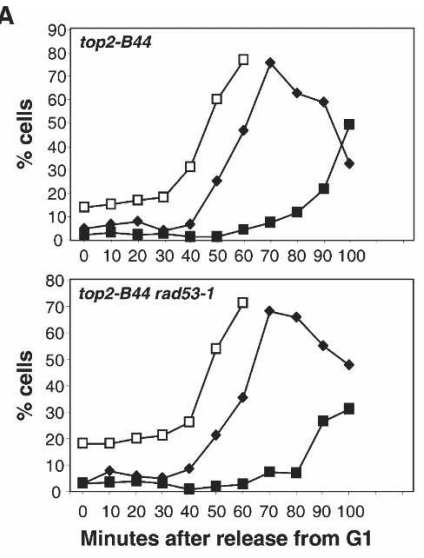

B

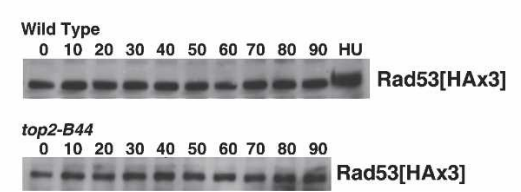

C

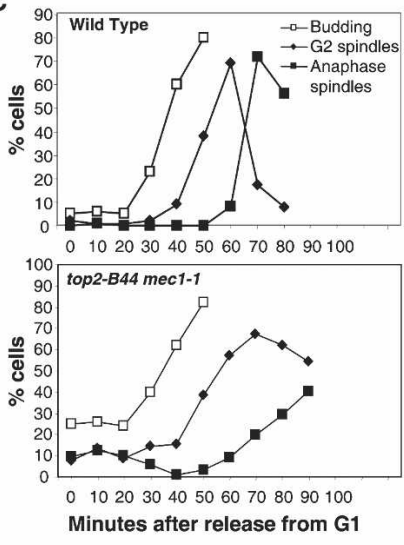

D

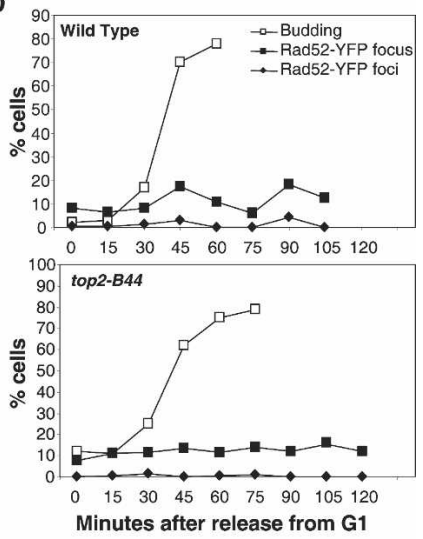

Figure 2. G2/M delay in top2-B44 is DNA damage checkpoint-independent. $(A, C)$ Cell cycle analysis of double mutants of top2::KAN pCEN-top2-B44 combined with DNA damage checkpoint mutants, performed as described in Figure 1 after G1 synchrony. $(A)$ Cell cycle progression after release from G1 in top2-B44 rad53-1. (C) Cell cycle progression after release from G1 in top2-B44 mec1-1 sml1s. (B) Western blot showing Rad53 phosphorylation shift after hydroxyurea (HU) treatment, but no shift in wild-type or top2-B44 cells progressing through the cell cycle at $32^{\circ} \mathrm{C}$, released from mating pheromone-induced G1 synchrony. $(D)$ Rad52 foci (a measure of the presence of DNA breaks) in wild-type or top2-B44 cells progressing through the cell cycle at $32^{\circ} \mathrm{C}$, released from mating pheromoneinduced G1 synchrony. Foci = cells with more than one fluorescent dot; focus = cells with one fluorescent dot.

low levels in wild-type and top2-B44 cells as they progressed through the cell cycle at $32^{\circ} \mathrm{C}$, indicating that the top2 mutant allele did not induce DNA damage as a consequence of its functional impairment (Fig. 2D). Some foci were expected to appear as cells progressed through S phase, based on previous work (Lisby et al. 2003). Considering each of these experiments, we conclude that dsDNA breaks are not frequently induced in top2-B44 cells and that the G2/M delay in these cells is not due to DNA damage checkpoint activation.

\section{G2/M delay in top2-B44 does not depend on Swe1}

In budding yeast, there are two known mechanisms that regulate onset of nuclear division. The first relies on Swel kinase, which negatively regulates Cdc28, the budding yeast cyclin-dependent kinase (Cdk). Secondly, the anaphase inhibitor, securin Pds1, can directly prevent anaphase onset. We had noticed that Cdc28 became modestly phosphorylated on Y19 in top2-B44 cells during the extended G2/M period (Supplementary Fig. 3), indicating a possible function of Swel in enforcing the G2/M delay. However, the length of the G2/M phase in top2-B44 swe1 $1 \Delta$ cells was indistinguishable from that of the top2-B44 single mutant (Fig. 3). Similarly, overexpression of Mihl, a phosphatase that counteracts Swe1dependent phosphorylation of Cdc28, did not shorten the G2/M-phase period in top2-B44 cells (Fig. 3). We conclude that negative regulation of $\mathrm{Cdc} 28$, at least through a known mechanism, is unlikely to produce the G2/M delay in top2-B44 cells.

\section{G2/M delay in top2-B44 depends on spindle checkpoint components}

We describe the cell cycle effect in top2-B44 cells as a G2/M-phase delay because this cycle phase in budding yeast is somewhat equivalent to metaphase in other eukaryotes (the mitotic spindle has assembled and the chromosomes have become bioriented on the spindle). A mammalian Topo II-dependent checkpoint has been argued to delay cells in metaphase-one report attributing this drug-induced delay to the presence of DNA damage (Mikhailov et al. 2002) and a conflicting study concluding that DNA damage is not the cause of the delay (Skoufias et al. 2004). In both instances, however, there was no evidence of spindle damage or a lack of chromosome attachment to the mitotic spindle that might have triggered the spindle-assembly checkpoint. Nevertheless, the delay in mammalian cells was at least partly bypassed in the absence of Mad2 (Mikhailov et al. 2002; Skoufias et al. 2004). These studies suggest that a Topo II-sensitive checkpoint might delay mammalian cells in metaphase rather than G2. We therefore tested whether the spindle checkpoint component Mad2 is required for the G2/M delay in top2-B44 cells. As shown in Figure 3, top2-B44 mad2 $\Delta$ cells budded and performed spindle assembly similar to wild-type cells. The G2/M delay seen in top2-B44 was completely bypassed, however, by the deletion of $M A D 2$, indicating that $\mathrm{Mad} 2$ is a component of the checkpoint system that induced a G2/M delay as a consequence of limited Topo II function. Deletion of Mad1, which forms a complex with Mad2, had a similar effect (Fig. 3), as did other spindle checkpoint compo- 
Andrews et al.

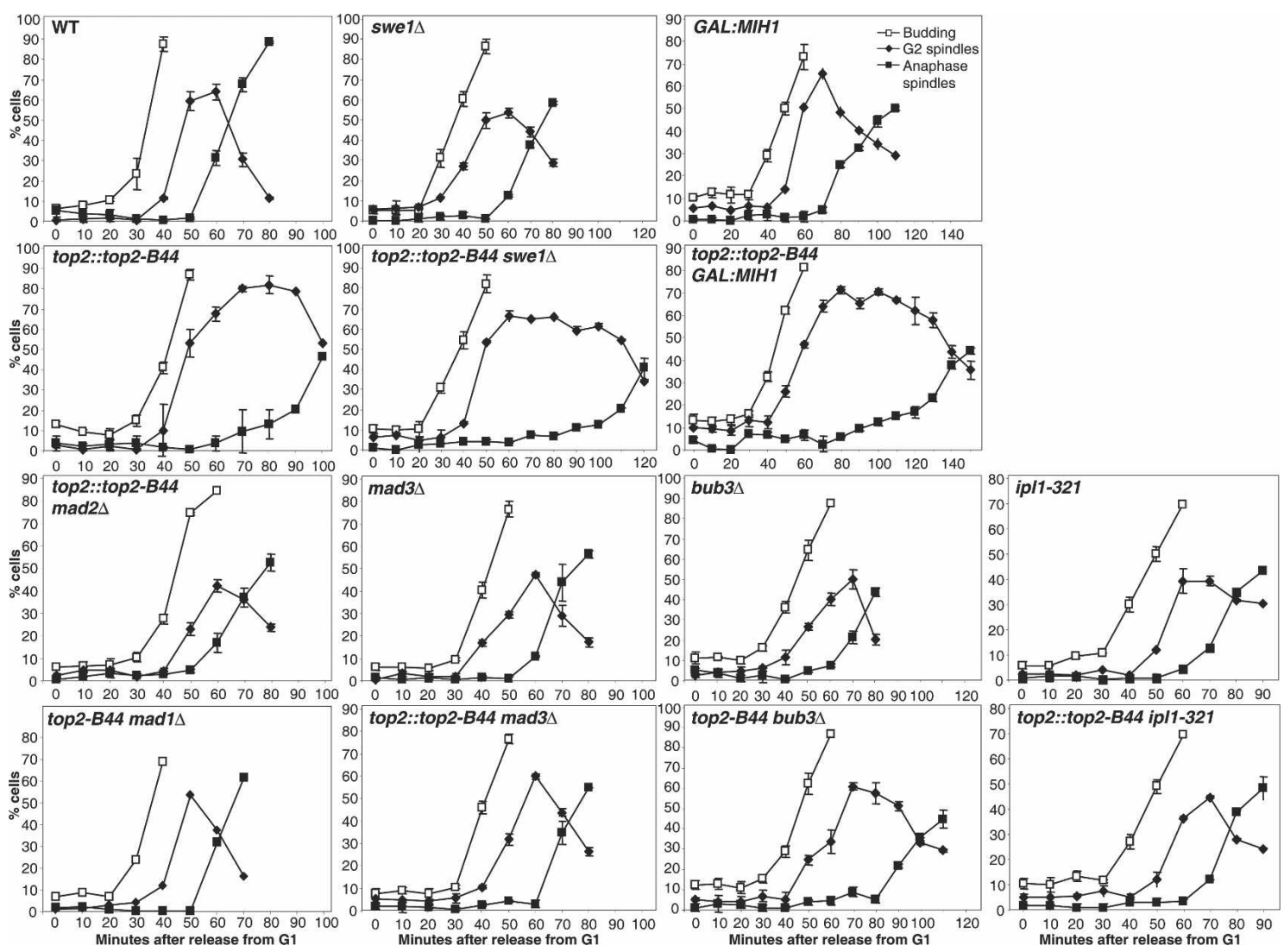

Figure 3. G2/M delay in top2-B44 depends on spindle checkpoint proteins, but not the Cdc28 kinase Swe1. Cell cycle analysis of double mutants of top 2::top2-B44 combined with a swe1-null or spindle assembly checkpoint mutants, performed as described in Figure 1 after G1 synchrony. In the case of the GAL1-MIH1 and top2::top2-B44 GAL1-MIH1 strains, the cells were synchronized in G1 in medium containing raffinose, then released into the cell cycle in the presence of galactose to induce overexpression of MIH1. For each time point, at least 200 cells were scored. The averages of several counts are plotted and the error bars show standard deviations. Derived from these data, approximate lengths of the G2/M period are listed for these stains in Supplementary Table 1.

nents, Mad3 and Ipl1. The spindle checkpoint protein Bub3 appeared to be partially required for the G2/M delay, since in numerous experiments, top2-B44 bub3s cells always had a G2/M period that was extended compared with wild-type cells, but that was shorter than top2-B44 cells (Fig. 3). Unfortunately, we were unable to determine whether Bub1 was required for the G2/M delay, because top2-B44 bub1s cells were extremely sick and could not be synchronized efficiently (data not shown). Bub2, a component of the MEN pathway that regulates exit from mitosis and responds to defects in spindle orientation (Gardner and Burke 2000; Poddar et al. 2004), was not required for the G2/M delay (data not shown).

Having identified spindle checkpoint proteins needed for the G2/M delay in top2-B44 cells, it is appropriate to describe this as a checkpoint-dependent delay, based on the classical genetic definition (Weinert and Hartwell 1988). It was equally important to determine whether the checkpoint is biologically relevant; i.e., whether this Topo II-dependent checkpoint enhances cell viability under conditions of limited Topo II function. We therefore compared the temperature sensitivities of top2-B44, top2-B44 mad2s, and top2-B44 mad1s cells growing on solid medium or after transient growth at high temperature in liquid medium (Supplementary Fig. 4A,B). In each case, viability was reduced when $M A D 1$ or $M A D 2$ were absent, indicating that the ability to execute the checkpoint delay in G2/M is important for cell survival.

When cell cycle checkpoints are bypassed, either using chemical inhibitors of checkpoint proteins or by genetic deletion of an essential checkpoint component, information about the function of the checkpoint system is often revealed. However, the rapid loss of cell viability in top2B44 mad2s and top2-B44 mad1s mutants could have stemmed from one of a number of cell cycle defects. If cell death in these mutants was linked to inappropriate anaphase onset in the presence of persistent DNA catenations, then aberrant chromosome segregation would be expected to occur, resulting in aneuploidy. To assay for unequal chromosome segregation, we examined cells prepared for FACScan analysis after a transient shift to the nonpermissive temperature. In wild-type cells and single mutants (top2-B44, mad1s, or mad2A), this treatment did not result in cells containing $<1 \mathrm{C}$ DNA content (Supplementary Fig. 4C). Strikingly, however, aneuploid $(<1 C)$ double-mutant top2-B44 mad2s and top2-B44 mad1s cells appeared after only $3 \mathrm{~h}$ at the 
nonpermissive temperature (about two cell division cycle times). Consistent with these data, DAPI staining revealed that nuclear DNA of top2- $B 44$ mad2 $\Delta$ cells, but not of top2-B44 cells, partitioned unequally during anaphase (Supplementary Fig. 4D). We conclude that perturbed Topo II function, in combination with a Topo II checkpoint defect, results in aneuploidy and cell death. Therefore, the Topo II checkpoint serves to protect from genome damage.

\section{G2/M checkpoint in top2-B44 is not enforced by $P d s 1 /$ securin}

The only known target of the spindle checkpoint pathway is the anaphase inhibitor Pds1/securin. Thus, we expected that a lack of Pds1/securin would abolish the ability of top2-B44 cells to delay in G2/M. However, after release from G1 synchrony of top2-B44 and top2-B44 pds $1 \Delta$, both strains delayed in G2/M to the same extent (Fig. 4). The length of the G2/M delay in these strains was highly reproducible, indicating that the lack of Pds1 did not even partially bypass the G2/M delay (Supple- mentary Fig. 5). Short and elongated spindles in all of the strains compared in these experiments were of similar lengths, suggesting that these parameters accurately represented G2/M phase and anaphase, respectively (Supplementary Fig. 5). Consistent with these experiments, G2/M delays were observed when top2-B44 cells were compared with top2-B44 pds1-128, possessing a checkpoint-null pds1 allele (Clarke et al. 1999; see Supplementary Figs. 6, 7). Importantly, the G2/M delay in top2-B44 pds1s and top2-B44 pds1-128 was not due to the lack of Pds1/securin, since $p d s 1-128$ and $p d s 1 \Delta$ single mutants progressed through G2/M with similar timing to wild-type cells (Fig. 4; Supplementary Fig. 6).

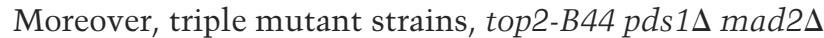
and top2-B44 pds1-128 mad2s, progressed through G2/M similar to wild type (Fig. 4; SupplementaryFig. 6), indicating that the perturbed Topo II function combined with the lack of Pds1 had not produced a deficiency that made the correct timing of anaphase onset inherently impossible.

A complication in the interpretation of the above analyses is that Pds1 not only inhibits Esp1 to prevent
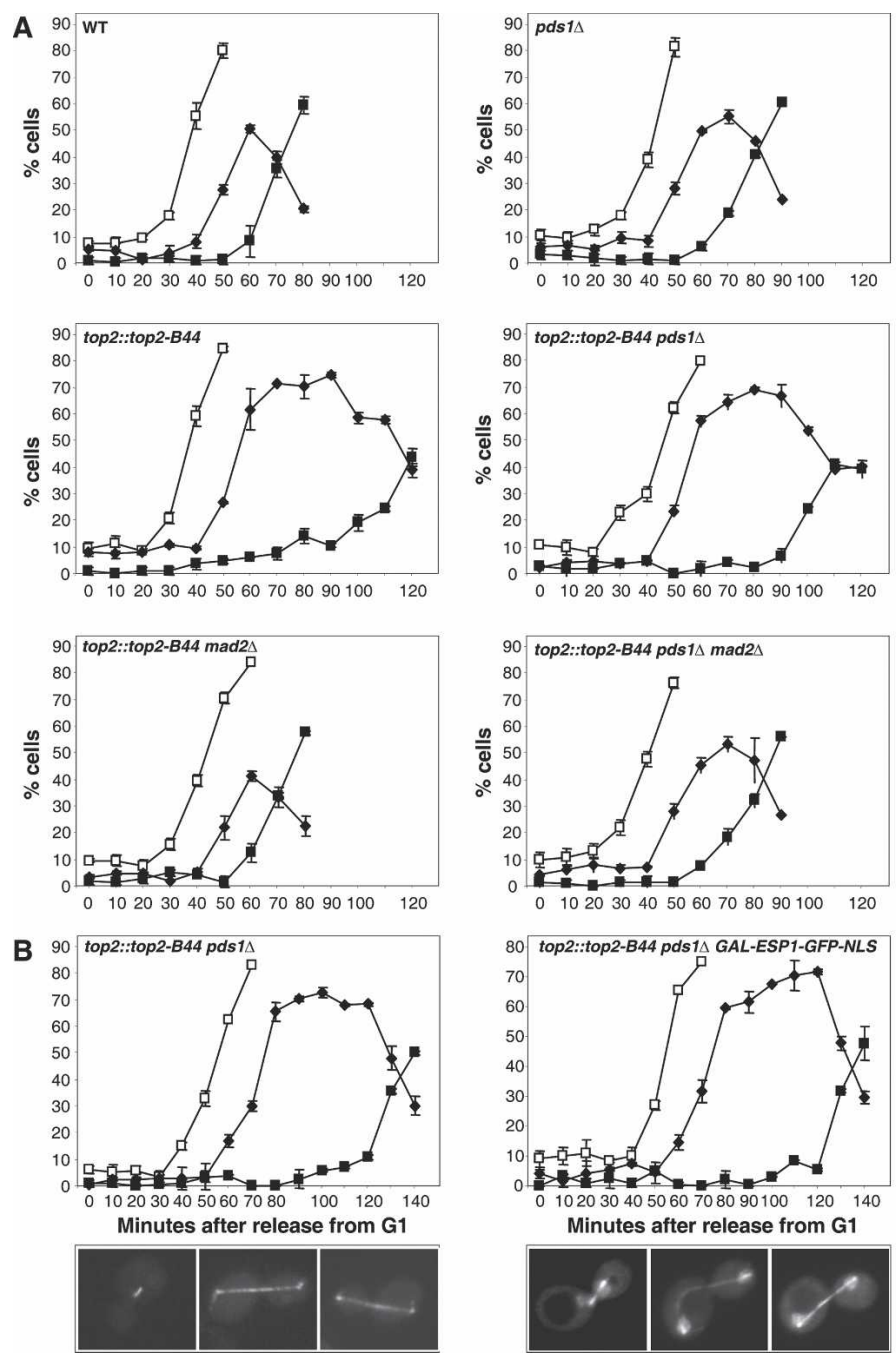

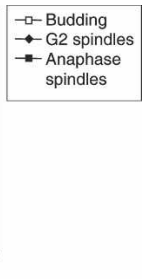

Figure 4. G2/M delay in top2-B44 is not enforced by Pds1/Securin. (A) Cell cycle analysis of double and triple mutants of top2::top2-B44 combined with $p d s 1 \Delta$ or $p d s 1 \Delta \operatorname{mad} 2 \Delta$. Since the $p d s 1 \Delta$ cells are temperature-sensitive slightly above $28^{\circ} \mathrm{C}$, the G1 synchrony was performed at $26^{\circ} \mathrm{C}$, then upon release from $\mathrm{G} 1$ the temperature was shifted up to $28^{\circ} \mathrm{C}$. (B) Cell cycle analysis of top 2::top2-B44 pds1s in combination with GAL1-ESP1-GFP-NLS (Jensen et al. 2001). Cells were synchronized in medium containing raffinose at $26^{\circ} \mathrm{C}$, then upon release from G1 the temperature was shifted up to $28^{\circ} \mathrm{C}$ and galactose was added to induce expression of Esp1-GFPNLS. (Note that the cell cycle progresses slightly slower in medium with raffinose/galactose as the carbon source.) As previously described (Jensen et al. 2001), accumulation of Esp1-GFP-NLS in the nucleus occurred efficiently in the majority of cells; photomicrographs show examples of G2/M and anaphase cells with nuclear Esp1 (right) and cells not expressing Esp1-GFP (left) for comparison. 
anaphase onset, but also aids in the nuclear import of Esp1, allowing Esp1 to access its targets. The possibility that G2/M delay in top2-B44 pds1s cells results from insufficient nuclear Esp1 must therefore be excluded. To address this concern, we compared the length of the G2/M period in top2-B44 pds $1 \Delta$ cells with the same cells overexpressing Esp1 that possessed an engineered nuclear localization signal (GAL1-ESP1-NLS) and had been previously shown to efficiently enter the nucleus in the absence of Pds1 (Jensen et al. 2001). Upon release from G1 synchrony, we were able to confirm efficient localization of Esp1-NLS to the nucleus, since the protein was also fused to GFP (Fig. 4). As a functional test for Esp1-NLS, we confirmed previous studies that demonstrated partial rescue of $p d s 1 \Delta$ temperature sensitivity (Jensen et al. 2001; data not shown). Importantly, the duration of G2/M phase was indistinguishable whether or not Esp1-NLS was overexpressed (Fig. 4) in top2-B44 pds1s cells.

Together, these experiments demonstrate that the checkpoint enforced by spindle checkpoint proteins, in response to perturbed Topo II function, cannot act via Pds1/securin. The budding yeast Topo II checkpoint must therefore be distinct from the spindle-assembly checkpoint, at least with respect to its target.

\section{G2/M delay in top2-B44 mutants depends on the anaphase-promoting complex (APC)}

That top2-B44 elicits a Pds1-independent pre-anaphase delay brings two important issues into question: first, whether inhibition of proteolysis by the APC is the mechanism of action of the Topo II checkpoint, and second, whether the delayed anaphase observed in top2-B44 cells indeed reflects anaphase onset in its normal cell cycle context. We began to address these questions by asking whether APC is inhibited during G2/M phase in top2-B44 cells. In this case, even though Pds1 is not required for the G2/M delay, we would expect subsequent events such as Pds1 degradation and nucleolar Cdc14 release to be delayed. First, we examined the kinetics of Cdc14 release from the nucleolus in wild-type and top2B44 cells (Supplementary Fig. 8). Consistent with previous work, partial Cdc14 release occurred coincident with the onset of spindle elongation in wild-type cells. In top2-B44 cells, partial Cdc14 release was substantially delayed, and in most cells, it did not occur until spindle elongation, although some G2/M top2-B44 cells were seen in which Cdc14 had become at least partially delocalized (Supplementary Fig. 8). Overall, however, these data indicated that the G2/M delay was accompanied by retention of Cdc14 in the nucleolus, and that Cdc14 release was coordinated with spindle elongation (though perhaps less efficiently than in the context of an unperturbed cell cycle).

We next examined Pds1 protein levels after release from G1 synchrony using an epitope-tagged (3xHA) version or Pds1. In wild-type and top2-B44 cells, some Pds1 was degraded 50-60 min after release from G1 synchrony (Fig. 5). Anaphase proceeded shortly thereafter in wild-

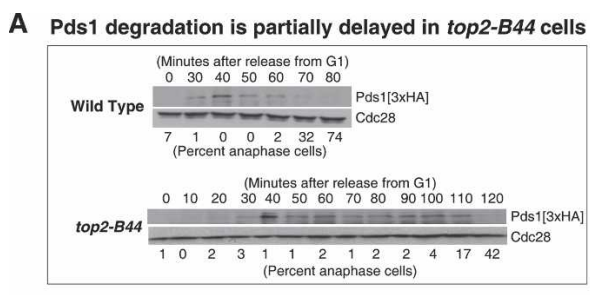

B Spindle elongation in top2-B44 cells depends on Apc2

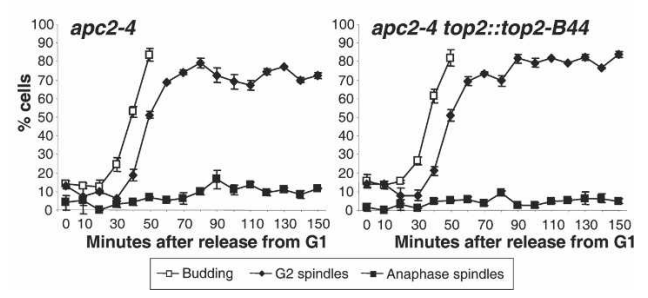

Figure 5. Spindle elongation in top2-B44 cells depends on the APC. (A) Timing of Pds1-3xHA degradation in top2-B44 cells. Cells were released from G1 synchrony as described in Figure 1 and samples were taken for scoring budding and spindle morphologies, as well as for preparing whole-cell protein extracts for Western blots. Pds1 was detected using an anti-HA antibody, and as a loading control, Cdc28 was detected using the PSTAIRE antibody. Percent anaphase cells at each time point is indicated beneath the Western blots. (B) Cell cycle analysis of top2::top2$B 44$ in combination with the apc2-4 mutation. Cells were synchronized in G1 at $26^{\circ} \mathrm{C}$, then upon release from G1 the temperature was shifted up to $32^{\circ} \mathrm{C}$, the nonpermissive temperature for the apc2-4 mutation. Budding and spindle morphologies were scored as described in Figure 1.

type cells and most of the remaining Pds1 was, as expected, degraded consistent with the timing of mitotic exit (data not shown). However, in top2-B44 cells, the remaining Pds1 protein (at $50 \mathrm{~min}$ ) stayed at a constant level until the time of anaphase, 110-120 min after release from G1. This indicated that with respect to its target Pds1, the APC was partially inhibited during G2/M in top2-B44 cells, and made it important to determine whether the APC was essential for spindle elongation in top2-B44 cells. To ask whether spindle elongation was completely dependent on the APC, we analyzed top2-B44 apc2-4 cells after release from G1 synchrony at the nonpermissive temperature for the apc2-4 mutation (Kramer et al. 1998). Spindle assembly in this strain occurred at a time similar to that in top2-B44 cells, but then these cells became blocked in G2/M phase with short spindles for at least $30 \mathrm{~min}$ after spindle elongation ought to have occurred (up to $150 \mathrm{~min}$ after G1 release; Fig. 5). This requirement for APC for spindle elongation in top2-B44 cells is consistent with the idea that the changes in microtubule dynamics leading to spindle elongation are indeed associated with anaphase onset. This is an important point to clarify since spindle elongation is not always APC-dependent and is not always indicative of anaphase onset (Clarke et al. 2003; Krishnan et al. 2004). We further evaluated this latter issue by examining loss of cohesion and segregation of a chromosome arm locus (LYS4) in wild-type and top2-B44 
cells. The timing of LYS4 segregation in top2-B44 cells was consistent with execution of a normal anaphase, after the G2/M delay (Supplementary Fig. 9). Based on all of these data-delayed Pds1 degradation, delayed Cdc14 release from the nucleolus, delayed LYS4 segregation, and the dependence of spindle elongation on the APCwe conclude that top2-B44 cells undergo an essentially normal anaphase after the G2/M-phase delay.

The simplest interpretation of these findings is that the G2/M delay in top2-B44 cells is enforced by inhibition of the APC by spindle assembly checkpoint proteins. We sought to determine whether Mad2-dependent inhibition of Cdc20 could account for APC inhibition during the G2/M delay in top2-B44 cells. We found that expression of a dominant allele of CDC20 (cdc20-50) (Schott and Hoyt 1998) that can activate the APC but is resistant to inactivation by $\mathrm{Mad} 2$ resulted in a partial bypass of the G2/M delay in top2-B44 cells (Supplementary Fig. 10). These data indicate that inhibition of Cdc20 by $\mathrm{Mad} 2$ at least partly accounts for inhibition of anaphase in top2-B44 cells. However, since only partial bypass was achieved, we cannot rule out the possibility that the spindle checkpoint acts through an additional mechanism to achieve APC inhibition. Nevertheless, all of these data suggest that APC inhibition leads to stabilization of an unknown anaphase inhibitor, other than Pds1, to enforce the G2/M delay.

\section{Chromosomes biorient properly in top2-B44 cells}

That Pds1/securin was not required for the G2/M delay in top2-B44 cells led us to question whether or not a conventional spindle checkpoint mechanism was triggered. The spindle checkpoint monitors kinetochoremicrotubule interactions, and thus the functional integrity of the spindle apparatus and kinetochores. When chromosomes are correctly bioriented, the spindle checkpoint is satisfied and Mad2-dependent inhibition of APC is reversed.

To ask whether top2-B44 cells are defective in satisfying the spindle checkpoint, we first compared the kinetics of chromosome biorientation in mutant and wildtype cells. In budding yeast, centromere DNA replication is completed well before anaphase (McCarroll and Fangman 1988); kinetochores can assemble and chromosomes are captured even while DNA replication is ongoing (Winey et al. 1995). Correct biorientation results in precocious centromere separation in budding yeast, resulting from the accurate attachment of a single microtubule from each spindle pole to each sister kinetochore in a bipolar arrangement, producing tension between the centromere regions (Fig. 6A; Goshima and Yanagida 2000). Analysis of the timing of precocious separation therefore measures kinetochore and spindle function as well as biorientation and tension. After release from G1 synchrony, wild-type and top2-B44 cells budded and initiated DNA synthesis after $\sim 30 \mathrm{~min}$ (Fig. 6B), the first cells reaching G2/M after $\sim 40-50 \mathrm{~min}$ in each case. Biorientation, as measured by precocious separation of a locus $1.2 \mathrm{~kb}$ away from CEN4, also occurred with similar tim- ing in the two strains. While anaphase, based on the segregation of this locus, occurred $\sim 12$ min after precocious separation in wild-type cells, it was not initiated in the top2-B44 cells until $\sim 35 \mathrm{~min}$ after precocious separation, consistent with the G2/M delay described above, based on spindle morphology. Thus, chromosomes seem to become bioriented with the correct timing in top2$B 44$ cells, and the spindle assembly checkpoint ought to have been satisfied. It is noteworthy, however, that this assay would be insensitive to more subtle defects in chromosome attachment.

top2-B44 cells do not delay after release from nocodazole-induced arrest

The spindle-assembly checkpoint monitors chromosome biorientation, and in response, controls the metaphase-to-anaphase transition. But the top2-B44 mutation delays cells prior to anaphase irrespective of correct chromosome biorientation. An inherent feature of the budding yeast cell cycle is that G2 phase and metaphase are not distinguishable cytologically. Since the spindle checkpoint ought to be satisfied in top2-B44 cells, we postulated that the cell cycle delay might in fact be a G2 delay triggered by reduced Topo II function. Alternatively, G2 and metaphase may indeed be indistinguishable in budding yeast, and the pre-anaphase delay seen in top2-B44 cells might be induced by slow decatenation rather than a kinetochore-spindle attachment defect. In either case, we would predict that top2-B44 cells ought to initiate anaphase spindle elongation with the same timing as wild-type cells when released from a nocodazole arrest. Our logic was to assume that nocodazole synchrony would either allow extra time for adequate resolution of the catenations or would allow the cells to progress beyond G2, after which the checkpoint would cease to function. To test these ideas, we arrested wildtype and top2-B44 cells in G1, with mating pheromone then released into the cell cycle at $32^{\circ} \mathrm{C}$ in the presence of nocodazole. Following release from $\mathrm{G} 1$, the cells reach G2/M after $\sim 50-60$ min (Figs. 1-5). We therefore released from the nocodazole synchrony after a further $60 \mathrm{~min}$, making the assumption that residual catenations would be resolved within this time frame and/or that the cells would progress beyond G2 phase. After removal of the nocodazole, wild-type and the top2-B44 cells assembled short spindles with similar timing. Anaphase was also initiated with similar kinetics in both strains, indicating that in agreement with the precocious CEN4 separation assay, chromosomes became attached to the spindle in a bipolar arrangement, and the spindle checkpoint was satisfied equally well in wild-type and top2-B44 cells (Fig. 6C).

We conclude that the likely cause of the cell cycle delay in top2-B44 cells is not a defect in spindle or kinetochore function, nor a defect in microtubule-kinetochore attachment, but rather a lack of Topo II function related to its decatenatory activity (Fig. 6D). This may help to explain why this checkpoint has a target other than Pds1. The extra time spent prior to anaphase (in the 
Andrews et al.

A

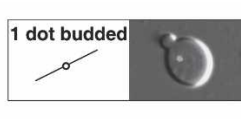

B
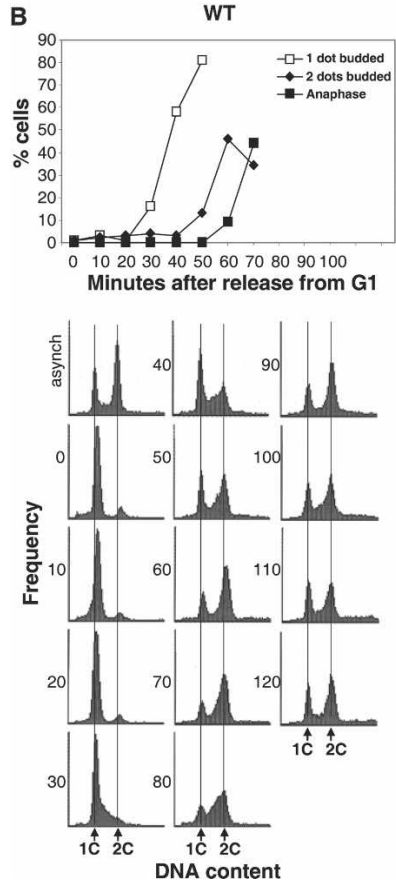
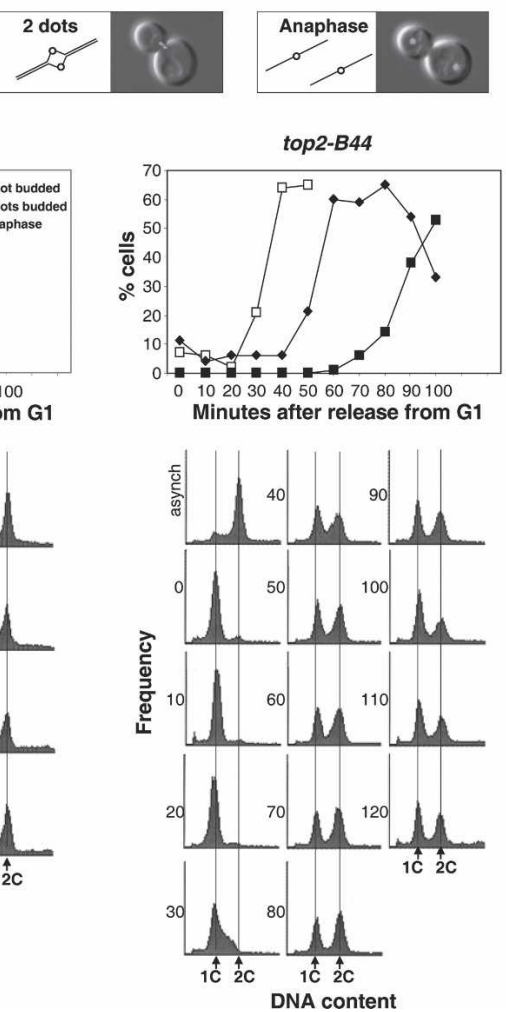

C
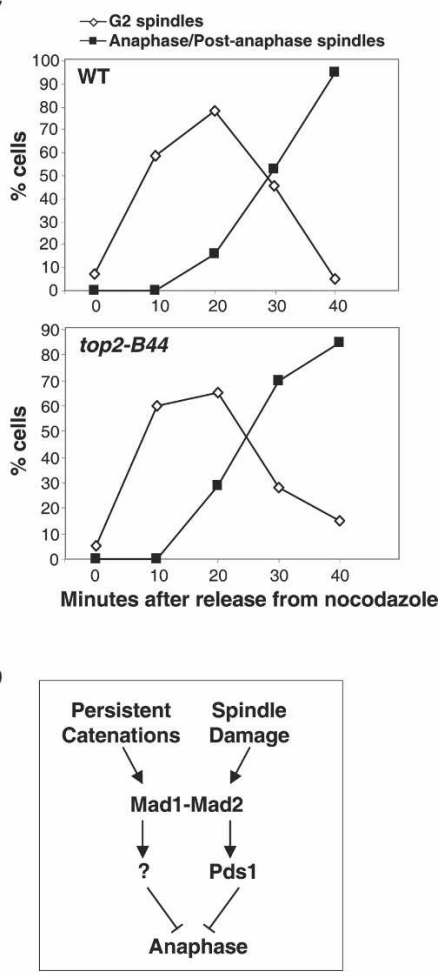

Figure 6. Chromosomes biorient properly in top2-B44 cells. (A) Schematic representation (cartoons) and photomicrographs describing a chromosome biorientation assay in budding yeast. (Left) A locus at CEN4 is tagged with a fluorescent signal (see Materials and Methods) yielding a single fluorescent dot in small budded cells. (Middle) Two closely apposed fluorescent spots are seen once the CEN4 locus has been replicated and has undergone the typical precocious separation (Goshima and Yanagida 2000) seen when the chromosome becomes bioriented, and is under spindle tension. (Right) During anaphase, the spots are segregated to the mother and daughter cells. $(B)$ Cell cycle analysis of wild-type and top2-B44 mutants after release from G1 synchrony. (Bottom) Cell morphologies were scored as depicted in $A$, and samples were processed for FACScan analysis of DNA content (time points are shown at the side of each histogram plot and the vertical lines indicate approximate position of 1C and 2C DNA contents). (C) Cell cycle analysis of wild-type versus top2-B44 mutants at $32^{\circ} \mathrm{C}$. Cells were released from mating pheromone-induced G1 synchrony into medium containing nocodazole, then released from the nocodazole after $120 \mathrm{~min}$. Samples were processed for cytology and spindle morphology was scored. (D) Model showing the proposed relationship between the Topo II checkpoint and the spindle assembly checkpoint in yeast. We suggest that the Topo II checkpoint is activated until decatenation reactions have been performed adequately to allow a successful mitosis. In this case, anaphase is inhibited independently of Pds1/securin.

presence of nocodazole) might have allowed the cells to progress beyond the cell cycle stage in which the checkpoint was operational (presumably G2) or could have simply allowed extra time for decatenation to occur, thus neutralizing the cause of checkpoint activation.

\section{Discussion}

The failure of Topo II checkpoint controls is likely to result in genome damage and genome instability (Trigueros and Roca 2001). Moreover, Topo II-dependent checkpoint defects were found in a subset of human cancers (Nakagawa et al. 2004), raising the possibility that such checkpoint defects could contribute to cancer etiology. An important study found that in some instances, Topo II-dependent checkpoint arrest led to mitotic cell death. Checkpoint-incompetent cells that were challenged with a chemotherapeutic Topo II poison were re- sistant to mitotic cell death, indicating that somatic mutations that inactivate Topo II checkpoints in tumor cells might cause resistance to chemotherapeutics that target Topo II (Vogel et al. 2005).

We report here the first description of a Topo II-dependent checkpoint in a genetically amenable eukaryote, budding yeast. This checkpoint apparently escaped previous detection because the commonly used top2-4 temperature-sensitive allele of TOP2 does not induce activation of this checkpoint pathway. We isolated new top2 alleles, most of which activate a G2/M checkpoint control, and we have characterized one such allele in detail (top2-B44). We provide evidence that DNA damage checkpoint components are not needed to enforce this checkpoint system that is triggered by perturbed Topo II function, and define in genetic terms several components of this pathway. Surprisingly, the yeast anaphase inhibitor Pds1/securin is not the checkpoint target. Nev- 
ertheless, a lack of this novel cell cycle checkpoint under conditions of perturbed Topo II function results in aneuploidy and cell death.

Previous work in mammalian cells relied on the use of inhibitors to study functions of Topo II in vivo. Commonly used drugs such as etoposide bind to Topo II either before or after passage of the T-strand, to form what is known as a "cleavable complex" (Zwelling et al. 1991). The topoisomerase II-mediated religation reaction, the last step of decatenation, is prevented and ultimately dsDNA breaks result. DNA damage affects cell cycle progression by inactivating p34 ${ }^{\mathrm{cdc} 2}-$ cyclin B1 complexes (Lock and Ross 1990), the kinase activity responsible for inducing entry into mitosis. Even the more recently used Topo II poisons that apparently do not cause gross chromosome damage, the bisdioxopiperazines (e.g., ICRF193), have been reported to induce DNA breaks indirectly as a consequence of Topo II dysfunction (Mikhailov et al. 2002). Thus, it has been difficult to make firm conclusions as to whether insufficient decatenation per se activates a checkpoint control independent of DNA damage that could be a consequence of persistent catenation. Using a yeast genetic system, however, we resolved this issue by demonstrating checkpoint activation in top2-B44 mutants that does not require key DNA damage checkpoint components such as Rad53, Mec1, and Pds1 (although we note that there are other DNA damage-responsive checkpoint kinases in yeast cells). We cannot rule out the possibility that DNA damage checkpoint controls are stimulated to some degree in top2-B44 cells because a minor population of (presumably) phosphorylated Rad53 was detectable on Western blots (see Fig. 2). However, we were unable to detect an increase in Rad52-containing nuclear foci in top2-B44 cells, indicating that double-stranded breaks were not more frequent than in wild-type cells.

While conflicting studies disagree over whether Topo II poisons such as ICRF-193 cause DNA damage, they similarly attest that the induced metaphase delay requires Mad2, an inhibitor of APC/C $\mathrm{Cdc}^{\mathrm{CO}}$. In mammals, Mad2 and Bub1 locate to kinetochores when the spindle checkpoint is activated (for review, see Nasmyth 2002), but photon counting studies have shown that this does not occur when the Topo II-dependent checkpoint is activated in metaphase (Skoufias et al. 2004). Spindle tension in metaphase, measured by the interkinetochore distance after Topo II inhibition, was indistinguishable from control metaphase cells (Skoufias et al. 2004). Conversely, defective cohesion that leads to chromosome misorientation and spindle checkpoint activation can be corrected by residual catenations maintained by Topo II inhibition (Vagnarelli et al. 2004). This correction not only restored sister association, but presumably also biorientation and tension, because the spindle checkpoint became silenced. Together, these studies indicate that inhibition of Topo II during mammalian mitosis does not activate the spindle checkpoint. Despite this, genetic studies using yeast have revealed that the sumoylation state of Top2 has important consequences within centric chromatin perhaps related to kinetochore structure/function (Bachant et al. 2002). This suggests that altered Top2 sumoylation might activate the spindle checkpoint due to the functional impairment of kinetochores or centromeric chromatin. Nonsumoylatable top2 mutants (top2-SNM) progress through G2/M with wild-type cell cycle kinetics (J. Bachant, pers. comm.), arguing that a lack of Top2 sumoylation does not result in checkpoint activation. In addition, none of the top2 mutant alleles that we found to delay in G2/M had mutations in any of the Top2 lysine residues that become sumoylated (Supplementary Fig. 2). We have not, however, excluded the possibility that other sumoylation defects (such as increased Top2 sumoylation) might be involved in activating the $\mathrm{G} 2 / \mathrm{M}$ checkpoint in top2-B44 cells.

Here we have used genetic analyses in yeast to show that top 2 mutants delay G2/M progression without a defect in chromosome biorientation, indicating that spindle and kinetochore functions are not perturbed. Taken together, these observations indicate that Topo II inhibition cannot result in a defect that triggers the spindle checkpoint. Our data, however, show unequivocally that yeast spindle checkpoint components are required to enforce the Topo II checkpoint. Thus, the Topo II checkpoint is distinct from the spindle checkpoint, but utilizes these key anaphase regulators.

In ICRF-193-induced mammalian mitotic arrest, Mad2 is associated with Cdc20 and Pds1/securin is stable (Skoufias et al. 2004). This observation suggested that Mad2-dependent APC inactivation prevents Pds1/securin degradation, thereby inhibiting anaphase onset. However, we find that a lack of Pds1 does not bypass the G2/M delay in yeast top2-B44 cells. Thus, we have demonstrated that Pds1/securin, the only known target downstream of the Mad1-Mad2 pathway in yeast is dispensable for the Topo II checkpoint response. These data indicate that a novel anaphase restraint mechanism is invoked in top2-B44 cells.

The nature of the cellular process monitored by Topo II-dependent checkpoints has not been determined. This is because it has been difficult to distinguish Topo II activity per se from physical aspects of chromosome structure that are formed as a consequence of Topo II activity. Here we have ruled out the possibility that one indirect consequence of perturbed Topo II, DNA breaks, is monitored by the checkpoint. A question that remains is whether decreased Topo II activity itself or persistent DNA catenation are monitored by the checkpoint. We have found that mutant top2 alleles differ in their ability to activate the checkpoint (Fig. 1; Supplementary Fig. 2). Thus, by comparing the defects that these alleles possess, and with a more detailed understanding of the Topo II catalytic cycle, we may eventually be able to gain insight into this important question.

In summary, we have genetically defined a Topo II checkpoint in yeast and provided evidence that it is needed for cell viability and the maintenance of euploidy. The checkpoint mechanism relies on spindle checkpoint components, but is not activated by spindle damage or a lack of proper chromosome biorientation. 
Regulation of anaphase onset in budding yeast is thought to depend on the Esp1/separase inhibitor Pds1/securin. But, since the Topo II checkpoint has a novel target, we speculate that anaphase onset might be controlled by an alternative mechanism (Fig. 6D).

\section{Materials and methods}

Yeast strain and plasmid construction

All strains are derived from BF264-15 15DU: MATa ura3sns ade1 his2 leu2-3,112 trp1-1 ${ }^{a}$ (Richardson et al. 1989). The TOP2 gene and its promoter were cloned by colony PCR and the PCR product was inserted into YCp50(URA3) and pRS414(TRP1). The TOP2 ORF was deleted as described (Wach et al. 1994). Temperature-sensitive top2 alleles were produced by random PCR-based mutagenesis combined with the plasmid shuffle method as previously described (Jensen et al. 2001).

\section{Yeast strain genotypes}

For yeast strain genotypes, see Supplemental material.

\section{Yeast cell cycle experiments}

Cells were grown to OD 2.0-4.0 overnight in synthetic medium containing additional adenine. For synchrony, cells were diluted to OD 0.15 in rich medium with $\alpha$ factor (concentrations varying from 1:1000 to 1:5000 of a $1 \mathrm{mg} / \mathrm{mL}$ stock). After $2-3 \mathrm{~h}$, cells were monitored microscopically and experiments were typically performed only if G1 synchrony of $85 \%-100 \%$ was reached. $\alpha$ Factor was washed off with water and cells were released under experimental conditions. Nocodazole was used at $0.15 \mu \mathrm{g} / \mathrm{mL}$. At each time point and for each strain, at least 200 cells were scored for cytology and samples were taken for FACScan analysis as described previously (Clarke et al. 2001; Haase and Reed 2002) using Sytox green DNA stain (Molecular Probes, Inc.). The G2/M cell cycle interval was measured for wild-type cells and top2-B44 mutants by selecting time-course experiments that adhered to the following criteria: (1) the percentage of cells with elongated spindles at a single time point reached at least $35 \%$, and (2) the kinetics of spindle formation and spindle elongation were approximately parallel. Then, we measured the time interval between spindle formation and elongation at the $35 \%$ elongated spindles mark. Standard deviations of the mean of at least eight experimental determinations were calculated.

\section{Microscopy}

Spindle morphologies were visualized using TUB1-GFP (Straight et al. 1997) and centromeres and the LYS4 locus were detected using the lacO/lacR-GFP system (Straight et al. 1996). Fluorescence and DIC microscopy with Plan Apo 63×/1.4 and Alpha Plan Fluar 100×/1.45 objectives and a Zeiss Axio Plan II microscope were used for scoring and capturing images of live cells with a Zeiss Axiocam camera and Axiovision software.

\section{Biochemistry}

Protein extracts were prepared using the glass bead method in NP40 buffer (50 mM Tris- $\mathrm{HCl}$ at $\mathrm{pH} 7.5,250 \mathrm{mM} \mathrm{NaCl}, 0.1 \%$ NP40, $10 \mathrm{mM}$ sodium pyrophosphate, $5 \mathrm{mM}$ EDTA, $0.1 \mathrm{mM}$ orthovanadate, $1 \mathrm{mM}$ PMSF, $2 \mu \mathrm{g} / \mathrm{mL}$ aprotinin, $2 \mu \mathrm{g} / \mathrm{mL}$ pepstatin, and $1 \mu \mathrm{g} / \mathrm{mL}$ leupeptin) and Western blots were per- formed as previously described (Jensen et al. 2001) using the following antibodies: 1:10,000 dilution of anti-HA (Santa Cruz) and a 1:10,000 dilution of anti-PSTAIRE. Secondary antibody, HRP-conjugated goat anti-mouse (Pierce) was used at 1:5000 and Western Lightning Chemiluminescence Reagent Plus (Perkin Elmer Life Sciences) was used as per the manufacturer's instructions.

\section{Acknowledgments}

We thank R. Rothstein for the Rad52-YFP strain, C. Holm for the top2-4 strain, C. Wittenberg for the RAD53-3xHA construct, A. Hoyt for the cdc20-50 mutant, D. Lew for the swe1 and GAL1-MIH1 strains, S. Biggins for the ipl-321 strain, S. Jensen for the GAL1-ESP1-NLS strain, L. Johnson for the apc2-4 strain, R. Li and K. Lee for the Cdc14-GFP strain, and J. Bachant for the LYS4-LacO strain. This work was funded by NIH grant CA099033 (D.J.C.).

\section{References}

Adachi, Y., Luke, M., and Laemmli, U.K. 1991. Chromosome assembly in vitro: Topoisomerase II is required for condensation. Cell 64: 137-148.

Adachi, N., Iiizumi, S., So, S., and Koyama, H. 2004. Genetic evidence for involvement of two distinct nonhomologous end-joining pathways in repair of topoisomerase II-mediated DNA damage. Biochem. Biophys. Res. Commun. 318: 856861.

Bachant, J., Alcasabas, A., Blat, Y., Kleckner, N., and Elledge, S.J. 2002. The SUMO-1 isopeptidase Smt4 is linked to centromeric cohesion through SUMO-1 modification of DNA topoisomerase II. Mol. Cell 9: 1169-1182.

Charron, M. and Hancock, R. 1990. DNA topoisomerase II is required for formation of mitotic chromosomes in Chinese hamster ovary cells: Studies using the inhibitor $4^{\prime}$-demethylepipodophyllotoxin 9-(4,6-O-thenylidene- $\beta$-D-glucopyranoside). Biochemistry 29: 9531-9537.

Clarke, D.J. and Giménez-Abián, J.F. 2000. Checkpoints controlling mitosis. Bioessays 22: 351-363.

Clarke, D.J., Johnson, R.T., and Downes, C.S. 1993. Topoisomerase II inhibition prevents anaphase chromatid segregation in mammalian cells independently of the generation of DNA strand breaks. J. Cell Sci. 105: 563-569.

Clarke, D.J., Segal, M., Mondesert, G., and Reed, S.I. 1999. The Pds1 anaphase inhibitor and Mec1 kinase define distinct checkpoints coupling $S$ phase with mitosis in budding yeast. Curr. Biol. 9: 365-368.

Clarke, D.J., Segal, M., Jensen, S., and Reed, S.I. 2001. Mec1p regulates Pds1p levels in S phase: Complex coordination of DNA replication and mitosis. Nat. Cell Biol. 3: 619-627.

Clarke, D.J., Segal, M., Andrews, C.A., Rudyak, S.G., Jensen, S., Smith, K., and Reed, S.I. 2003. S-phase checkpoint controls mitosis via an APC-independent Cdc20p function. Nat. Cell Biol. 21: 21.

Cook, P.R. 1991. The nucleoskeleton and the topology of replication. Cell 66: 627-635.

Creighton, A.M. and Birnie, G.D. 1969. The effect of bisdioxopiperazines on the synthesis of deoxyribonucleic acid, ribonucleic acid and protein in growing mouse-embryo fibroblasts. Biochem. J. 114: $58 \mathrm{P}$.

Deming, P.B., Cistulli, C.A., Zhao, H., Graves, P.R., PiwnicaWorms, H., Paules, R.S., Downes, C.S., and Kaufmann, W.K. 2001. The human decatenation checkpoint. Proc. Natl. 
Acad. Sci. 98: 12044-12049.

DiNardo, S., Voelkel, K., and Sternglanz, R. 1984. DNA topoisomerase II mutant of Saccharomyces cerevisiae: Topoisomerase II is required for segregation of daughter molecules at the termination of DNA replication. Proc. Natl. Acad. Sci. 81: 2616-2620.

Dominguez, I., Pastor, N., Mateos, S., and Cortes, F. 2001. Testing the SCE mechanism with non-poisoning topoisomerase II inhibitors. Mutat. Res. 497: 71-79.

Downes, C.S., Clarke, D.J., Mullinger, A.M., Giménez-Abián, J.F., Creighton, A.M., and Johnson, R.T. 1994. A topoisomerase II-dependent G2 cycle checkpoint in mammalian cells. Nature 372: 467-470.

Earnshaw, W.C., Halligan, B., Cooke, C.A., Heck, M.M., and Liu, L.F. 1985. Topoisomerase II is a structural component of mitotic chromosome scaffolds. J. Cell Biol. 100: 1706-1715.

Gardner, R.D. and Burke, D.J. 2000. The spindle checkpoint: Two transitions, two pathways. Trends Cell Biol. 10: 154158.

Giménez-Abián, J.F. and Clarke, D.J. 2003. Replication-coupled topoisomerase II templates the mitotic chromosome scaffold? Cell Cycle 2: 230-232.

Giménez-Abián, J.F., Clarke, D.J., Mullinger, A.M., Downes, C.S., and Johnson, R.T. 1995. A postprophase topoisomerase II-dependent chromatid core separation step in the formation of metaphase chromosomes. J. Cell Biol. 131: 7-17.

Giménez-Abián, J.F., Clarke, D.J., Devlin, J., Giménez-Abián, M., De la Torre, C., Johnson, R.T., Mullinger, A.M., and Downes, C.S. 2000. Premitotic chromosome individualization in mammalian cells depends on topoisomerase II activity. Chromosoma 109: 235-244.

Gimenez-Abian, J.F., Weingartner, M., Binarova, P., Clarke, D.J., Anthony, R.G., Calderini, O., Heberle-Bors, E., Moreno Diaz de la Espina, S., Bogre, L., and De la Torre, C. 2002. A topoisomerase II-dependent checkpoint in G2-phase plant cells can be bypassed by ectopic expression of mitotic cyclin B2. Cell Cycle 1: 187-192.

Goshima, G. and Yanagida, M. 2000. Establishing biorientation occurs with precocious separation of the sister kinetochores, but not the arms, in the early spindle of budding yeast. Cell 100: 619-633.

Haase, S.B. and Reed, S.I. 2002. Improved flow cytometric analysis of the budding yeast cell cycle. Cell Cycle 1: 132-136.

Hajji, N., Pastor, N., Mateos, S., Dominguez, I., and Cortes, F. 2003. DNA strand breaks induced by the anti-topoisomerase II bis-dioxopiperazine ICRF-193. Mutat. Res. 530: 35-46.

Hirano, T. and Mitchison, T.J. 1991. Cell cycle control of higher-order chromatin assembly around naked DNA in vitro. J. Cell Biol. 115: 1479-1489.

Holm, C., Goto, T., Wang, J.C., and Botstein, D. 1985. DNA topoisomerase II is required at the time of mitosis in yeast. Cell 41: 553-563.

Jensen, S., Segal, M., Clarke, D.J., and Reed, S.I. 2001. A novel role of the budding yeast separin Esp1 in anaphase spindle elongation: Evidence that proper spindle association of Esp1 is regulated by Pds1. J. Cell Biol. 152: 27-40.

Kalwinsky, D.K., Look, A.T., Ducore, J., and Fridland, A. 1983. Effects of the epipodophyllotoxin VP-16-213 on cell cycle traverse, DNA synthesis, and DNA strand size in cultures of human leukemic lymphoblasts. Cancer Res. 43: 1592-1597.

Kramer, K.M., Fesquet, D., Johnson, A.L., and Johnston, L.H. 1998. Budding yeast RSI1/APC2, a novel gene necessary for initiation of anaphase, encodes an APC subunit. EMBO T. 17: 498-506.

Krishnan, V., Nirantar, S., Crasta, K., Cheng, A.Y., and Surana,
U. 2004. DNA replication checkpoint prevents precocious chromosome segregation by regulating spindle behavior. Mol. Cell 16: 687-700.

Lisby, M., Antunez de Mayolo, A., Mortensen, U.H., and Rothstein, R. 2003. Cell cycle-regulated centers of DNA doublestrand break repair. Cell Cycle 2: 479-483.

Lock, R.B. and Ross, W.E. 1990. Inhibition of p34cdc2 kinase activity by etoposide or irradiation as a mechanism of G2 arrest in Chinese hamster ovary cells. Cancer Res. 50: 37613766.

McCarroll, R.M. and Fangman, W.L. 1988. Time of replication of yeast centromeres and telomeres. Cell 54: 505-513.

Mikhailov, A., Cole, R.W., and Rieder, C.L. 2002. DNA damage during mitosis in human cells delays the metaphase/anaphase transition via the spindle-assembly checkpoint. Curr. Biol. 12: 1797-1806.

Mikhailov, A., Shinohara, M., and Rieder, C.L. 2004. Topoisomerase II and histone deacetylase inhibitors delay the G2/M transition by triggering the p38 MAPK checkpoint pathway. I. Cell Biol. 166: 517-526.

Munoz, P., Baus, F., and Piette, J. 2001. Ku antigen is required to relieve G2 arrest caused by inhibition of DNA topoisomerase II activity by the bisdioxopiperazine ICRF-193. Oncogene 20: 1990-1999.

Nakagawa, T., Hayashita, Y., Maeno, K., Masuda, A., Sugito, N., Osada, H., Yanagisawa, K., Ebi, H., Shimokata, K., and Takahashi, T. 2004. Identification of decatenation G2 checkpoint impairment independently of DNA damage G2 checkpoint in human lung cancer cell lines. Cancer Res. 64: 48264832.

Nasmyth, K. 2002. Segregating sister genomes: The molecular biology of chromosome separation. Science 297: 559-565.

Newport, J. 1987. Nuclear reconstitution in vitro: Stages of assembly around protein-free DNA. Cell 48: 205-217.

Poddar, A., Daniel, J.A., Daum, J.R., and Burke, D.J. 2004. Differential kinetochore requirements for establishment and maintenance of the spindle checkpoint are dependent on the mechanism of checkpoint activation in Saccharomyces cerevisiae. Cell Cycle 3: 197-204.

Richardson, H.E., Wittenberg, C., Cross, F.R., and Reed, S.I. 1989. An essential G1 function for cyclin-like proteins in yeast. Cell 59: 1127-1133.

Schott, E.J. and Hoyt, M.A. 1998. Dominant alleles of Saccharomyces cerevisiae CDC20 reveal its role in promoting anaphase. Genetics 148: 599-610.

Shamu, C.E. and Murray, A.W. 1992. Sister chromatid separation in frog egg extracts requires DNA topoisomerase II activity during anaphase. J. Cell Biol. 117: 921-934.

Skoufias, D.A., Lacroix, F.B., Andreassen, P.R., Wilson, L., and Margolis, R.L. 2004. Inhibition of DNA decatenation, but not DNA damage, arrests cells at metaphase. Mol. Cell 15: 977-990.

Straight, A.F., Belmont, A.S., Robinett, C.C., and Murray, A.W 1996. GFP tagging of budding yeast chromosomes reveals that protein-protein interactions can mediate sister chromatid cohesion. Curr. Biol. 6: 1599-1608.

Straight, A.F., Marshall, W.F., Sedat, J.W., and Murray, A.W. 1997. Mitosis in living budding yeast: Anaphase A but no metaphase plate. Science 277: 574-578.

Sundin, O. and Varshavsky, A. 1981. Arrest of segregation leads to accumulation of highly intertwined catenated dimers: Dissection of the final stages of SV40 DNA replication. Cell 25: 659-669.

Tanabe, K., Ikegami, Y., Ishida, R., and Andoh, T. 1991. Inhibition of topoisomerase II by antitumor agents bis(2,6- dioxo- 
Andrews et al.

piperazine) derivatives. Cancer Res. 51: 4903-4908.

Trigueros, S. and Roca, J. 2001. Circular minichromosomes become highly recombinogenic in topoisomerase-deficient yeast cells. J. Biol. Chem. 276: 2243-2248.

Uemura, T. and Yanagida, M. 1984. Isolation of type I and II DNA topoisomerase mutants from fission yeast: Single and double mutants show different phenotypes in cell growth and chromatin organization. EMBO J. 3: 1737-1744.

Uemura, T., Morikawa, K., and Yanagida, M. 1986. The nucleotide sequence of the fission yeast DNA topoisomerase II gene: Structural and functional relationships to other DNA topoisomerases. EMBO J. 5: 2355-2361.

Vagnarelli, P., Morrison, C., Dodson, H., Sonoda, E., Takeda, S., and Earnshaw, W.C. 2004. Analysis of Scc1-deficient cells defines a key metaphase role of vertebrate cohesin in linking sister kinetochores. EMBO Rep. 5: 167-171.

Vogel, C., Kienitz, A., Muller, R., and Bastians, H. 2005. The mitotic spindle checkpoint is a critical determinant for topoisomerase-based chemotherapy. J. Biol. Chem. 280: 40254028.

Wach, A., Brachat, A., Pohlmann, R., and Philippsen, P. 1994. New heterologous modules for classical or PCR-based gene disruptions in Saccharomyces cerevisiae. Yeast 10: 17931808.

Wasserman, S.A. and Cozzarelli, N.R. 1986. Biochemical topology: Applications to DNA recombination and replication. Science 232: 951-960.

Weinert, T.A. and Hartwell, L.H. 1988. The RAD9 gene controls the cell cycle response to DNA damage in S. cerevisiae. Science 241: 317-322.

Winey, M., Mamay, C.L., O'Toole, E.T., Mastronarde, D.N., Giddings Jr., T.H., McDonald, K.L., and McIntosh, J.R. 1995. Three-dimensional ultrastructural analysis of the Saccharomyces cerevisiae mitotic spindle. J. Cell Biol. 129: 16011615.

Wood, E.R. and Earnshaw, W.C. 1990. Mitotic chromatin condensation in vitro using somatic cell extracts and nuclei with variable levels of endogenous topoisomerase II. J. Cell Biol. 111: 2839-2850.

Yanagida, M. and Wang, J.C. 1987. Yeast DNA topoisomerases and their structural genes. In Nucleic acids and molecular biology (eds. F. Eckstein and D.M.J. Lilley), pp. 196-209. Springer-Verlag, Berlin and Heidelberg, Germany.

Zwelling, L.A., Mayes, J., Hinds, M., Chan, D., Altschuler, E., Carroll, B., Parker, E., Deisseroth, K., Radcliffe, A., Seligman, M., et al. 1991. Cross-resistance of an amsacrine-resistant human leukemia line to topoisomerase II reactive DNA intercalating agents. Evidence for two topoisomerase II directed drug actions. Biochemistry 30: 4048-4055. 


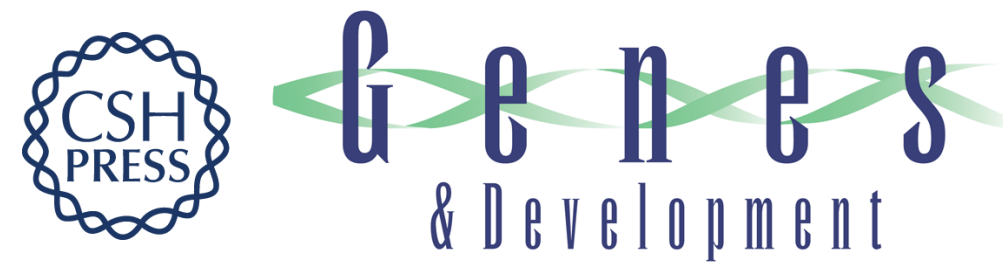

\section{A mitotic topoisomerase II checkpoint in budding yeast is required for genome stability but acts independently of Pds1/securin}

Catherine A. Andrews, Amit C. Vas, Brian Meier, et al.

Genes Dev. 2006, 20:

Access the most recent version at doi:10.1101/gad.1367206

Supplemental http://genesdev.cshlp.org/content/suppl/2006/04/17/20.9.1162.DC1
Material

References This article cites 59 articles, 24 of which can be accessed free at: http://genesdev.cshlp.org/content/20/9/1162.full.html\#ref-list-1

License

Email Alerting Receive free email alerts when new articles cite this article - sign up in the box at the top Service right corner of the article or click here.

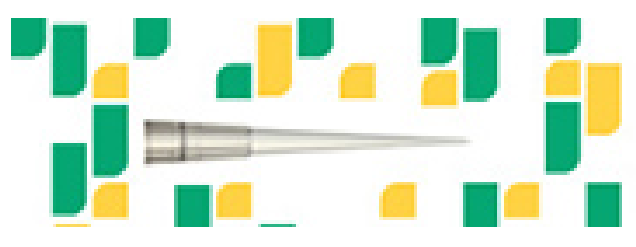

Focused on your science. 